\title{
What are local issues? The problem of the local review of research
}

\author{
P Wainwright, J Saunders
}

Local issues should be addressed through the governance framework and not through the research ethics committee system

l has been widely accepted in the past that the ethical review of research should be carried out by a body with some responsibility for, and knowledge of, the local community or population within which the research is to be performed. In the UK the historical development of NHS research ethics committees (RECs) has taken place in the context of local health service organisational structure, with local RECs being created and administered as a function of the local health authority. Traditionally this local responsibility has been fiercely guarded, with much being made of issues of independence and autonomy on the part of local committees to allow or disallow research in their territory on their own terms. From quite an early stage, Department of Health ${ }^{1}$ guidelines made provision for the delegation of responsibility from one committee to another:

Multicentre research: 2.18 "Each LREC is free to arrive at its own decision when considering a proposal which is planned to take place in more than one area. It would, however, obviously be sensible-in the interests of eliminating unnecessary delay and of ensuring that similar criteria are used to consider a proposal-that committees should arrive at a voluntary arrangement under which one LREC is nominated to consider the issue on behalf of them all. Health Authorities should positively encourage networks for neighbouring LRECs so that such cooperation is more easily achieved" (DoH 1991).

However, although this was intended to apply to studies taking place in more than one health authority's area, for example in multicentre studies, in practice such cooperative arrangements rarely occurred. Indeed, the multiple review of multicentre studies became so problematic that the Centre for Philosophy and Health Care at the University of Wales Swansea was commissioned to review the situation, ${ }^{2}$ and in due course the Multi Centre Research Ethics Committee structure was created. $^{3}$

\section{Abstract}

Local review of research by ethics committees in the UK has long been held to be an important right of the local research ethics committee and, even with the introduction of the European Clinical Trials Directive, the governance arrangements for research ethics committees continue to allow for local review of multicentre studies. There is no requirement for local review in either the European Union directive or in the guidelines on good clinical practice, and there is little evidence of it anywhere else in Europe. The idea that there can be "local", as opposed to "central" ethical issues in research is an interesting one, which raises important issues about the nature of research ethics and ethical review. The aim of this paper is to argue that there are no such things as local issues in research ethics, and suggest that those questions currently addressed as local issues properly belong within the research governance framework.

Even within the MREC-LREC system the right of local committees to review multicentre studies for "local issues" was retained and, from the perspective of the intended cooperative working arrangements set out in the 1991 guidance, it could be argued that "local ethical issues" were invented at this point. The recent European Clinical Trials Directive ${ }^{4}$ seeks to streamline the process of review and requires that "Member States shall establish a procedure providing, notwithstanding the number of Ethics Committees, for the adoption of a single opinion for that Member State". The directive also stipulates that the ethics committee "shall consider", among other things, "the suitability of the investigator and supporting staff" and "the quality of the facilities" although there is no mention in the directive of this being done at any specifically local level, or other than as part of the single opinion for the Member State.

The Department of Health Governance Arrangements for Research Ethics Committees (GAFREC) ${ }^{5}$ sets out the framework for review of locality issues, which are limited to:

- the suitability of the local researcher

- the appropriateness of the local research environment and facilities

- specific issues relating to the local community, including the need for provision of information in languages other than English.
The role of the research ethics committee is also discussed in the latest draft of the guidelines on good clinical practice (GCP). ${ }^{6}$ These guidelines state that: "One of the responsibilities of the Ethics Committee is to safeguard the rights, safety and wellbeing of all trial subjects and to provide public assurance of that protection by, among other things, expressing an opinion on the trial protocol, the suitability of the investigators and the adequacy of the facilities, and on the methods and documents to be used to inform trial subjects and obtain their informed consent. Special attention should be paid to trials that may include vulnerable subjects."

However, there is once again no specific reference to local review, only the requirement that "Member States shall establish a procedure for the adoption of a single Ethics Committee opinion for each Member State". In providing for the possibility of the local review of multicentre studies in the UK, the DoH in GAFREC currently does appear to be going beyond the requirements of the European directive and of the GCP guidance.

It is, of course, entirely possible for different individuals, acting from

Abbreviations: EU, European Union; GAFREC, governance arrangements for research ethics committees; GCP, good clinical practice; REC, research ethics committee 
sound moral principles, to come to different conclusions about the ethical acceptability of any given research study. However, there is an inherent tension in the idea that there can be local ethical issues (as opposed, one must presume, to "central ethical issues") that render a study ethically sound in one place and not in another. The intention in this article is to argue that there are no such things as local ethical issues and that the review of matters currently described as "locality issues" should not be the responsibility of a research ethics committee.

\section{ARE LOCAL ISSUES ETHICAL IN NATURE?}

The ethical review of research must address several issues. These include, but may not be confined to, the safety and protection of the vulnerable human participant, questions of equipoise and the value of the research, the appropriateness of the methods and balance of risk and benefit, and the arrangements for a proper, informed consent by participants who have the capacity to give consent. These issues are mostly determined when a study is in the design stage. Investigators must be able to justify the research on the grounds that there is a worthwhile question, to which we do not know the answer. They must be convinced that the question is capable of being answered, in ways that do not involve unacceptable danger to participants, and must create a design that makes appropriate use of methods such as randomisation, blinding, placebo, takes into account availability of other treatments for the condition in question, is based on an adequate sample with power to detect a significant difference in outcome, and so on. They then have to produce an explanation of the study and all its implications that is comprehensible to all likely participants. In doing this they should have in mind the probable age and mental status of participants, and the possible likelihood of involvement of participants from varied ethnic or cultural backgrounds. These questions will have scientific importance, as they will impact upon the homogeneity of the sample and the introduction of confounding variables. They will also be of ethical significance and will be the issues that a lead or main ethics committee will consider. The ethical acceptability of the study design or protocol will not, however, vary in virtue of the locality in which the research is to take place. We may disagree about the use of placebo, the additional battery of tests over and above normal treatment, the risks that arise from the withdrawal of standard treatment, or the acceptability of the use of deception, but if we find these things acceptable or unacceptable we should be of the same opinion regardless of the area in which the study is to take place.

Local issues as implied by the European Union (EU) directive and set out explicitly in GAFREC, are confined to the suitability of the local researcher, the appropriateness of the local research environment and facilities, and specific issues relating to the local community, including the need for provision of information in languages other than English. It would of course be unethical to allow an incompetent investigator to run a clinical trial at a particular centre. It would also be unethical to present the local ethnic community, many of whom do not speak English, with information and consent materials printed only in English, or to expect an English speaking child of the family to interpret these materials for the non-English speaking parents. But it would be equally unethical for the local NHS trust or general practice to allow an incompetent clinician to have responsibility for patient care, or to present members of the local ethnic community with surgical consent forms printed only in English and to expect the child to interpret information about surgery or other treatment for the parent. These are not questions of research ethics, they are simply bad things to do. They are of course unethical, but the ethical standard is one that is set at the national or international level, and not at the local.

This argument is supported by procedures that have been in place for some time to deal with epidemiological research and other types of study in which there is no local researcher. These guidelines, issued by COREC in November $2000,{ }^{7}$ observe that in many types of study there is no need for there to be a local investigator, even in some instances in which there is patient contact by a local clinician and the collection of data including tissue samples. The guidelines note that "Many LRECs themselves have questioned the need for a local REC opinion in these cases, as long as sufficient safeguards are confirmed to be in place during the ethical review of the protocol by another REC in the NHS". The guidelines therefore establish the principle that if there is no local investigator, there is no need for LREC approval and thus, we must assume, no local issues. All questions regarding the ethical conduct of the research are addressed by a single, central review and any local committees are simply notified for information.

If the key factor determining whether or not a study needs local review is the presence or not of a local investigator, and it is accepted that all other ethical questions about the conduct of the study can be addressed centrally, this would seem to support our argument that all research ethics questions of design, method, information, consent, patient safety, and so on, can be reviewed centrally by one committee. None of these matters rest with the local investigator. The only matters that could be said to rest with the local investigator are those things that he or she brings to the study, which is to say his or her expertise, conduct, and competence, and any facilities or resources he or she may be required to provide. The question for local review thus is not whether the study is ethical, but whether the local investigator is a fit person, with adequate facilities. And these questions, of course, are not ethical but empirical. It might be generally agreed for example that, to be an acceptable local investigator, a researcher should have an appropriate higher degree and some evidence of previous successful involvement in research, perhaps in the form of peer reviewed publications. The local question of the suitability of any specific investigator can then be answered by reading his curriculum vitae to look for empirical evidence of his qualifications and publication record.

In a recent survey, ${ }^{8}$ Megone and colleagues highlight the variation across the EU in the local responsibilities of RECs. Of those that are local they found two kinds: those that oversee a local area (as in the UK) or those that are institutionally based (for example a hospital committee). Apart from the UK, only Germany, Greece, and Spain had local RECs of the first kind and, like the UK, consequently have a large number of committees. A similar analysis emerges from a Council of Europe Survey, ${ }^{9}$ which says very little about the need for local review among the 28 countries that responded. In most countries (15 of 28) RECs were hospital or university based and in 13 they were based on professional bodies. Most ethical review of research within Europe therefore takes place without any perceived need to review "local" ethical factors.

\section{WHO SHOULD BE RESPONSIBLE FOR LOCALITY ISSUES?}

The assumption that underlies the system of local review by RECs is that, because of their local nature, members of an REC will have personal knowledge of their local investigators, local health services facilities, and the local health economy. This may have been true when RECs were first established 20 years ago, and may sometimes be true 
today. However, recent changes in the NHS in England and Wales have undermined this position. A Health Authority was a large entity, typically covering a population of around 200000 people, and employing around 30000 staff. Although the proportion of staff involved in research must be small, the dozen or so members of the REC (a proportion of whom would be lay) could not have been expected to have good personal knowledge of every local researcher. Where members did have personal knowledge, it was as likely to be because of a professional or social relationship with the individual, a situation that in today's climate would require at the very least a declaration of a possible conflict of interest.

The recent changes have required a redefinition of the research site, for the purposes of defining a single or multicentre study, in England by the creation of the Strategic Health Authority, and in Wales by the abolition of health authorities and the creation of local health boards. It is likely that in Wales the definition of a site will be by reference to three regional offices for the NHS in Wales. In both instances, the geographical area encompassed within a single centre study will be greater than that covered by the former health authorities. Because one committee will be required to consider a study taking place within one of these areas, the probability that REC members will have personal knowledge of the applicants' competence, facilities, and resources is accordingly diminished.

If researchers, who are likely to have considerable personal investment in the success of an application in financial, academic, or professional career terms (or all three), are to have their competence and suitability assessed by the REC, they are surely entitled to know by what standards and criteria, and on what evidence this assessment is to be made. Given the new legal status of the RECs, a disappointed applicant, rejected as unsuitable, would be entitled to seek judicial review of the decision and, given the present lack of standards or criteria, or any systematic process of assessment, one might suppose that the courts will take a dim view of the REC decision making process.

When participants are recruited to a study through an NHS organisation, or by virtue of their status as NHS patients, they are entitled to expect the relevant NHS organisation to accept responsibility for their well being in the same way as they expect to be properly treated as patients in receipt of care. The management responsibility in, for example, an NHS trust, will rest with the chief executive and the board of directors, but will usually be exercised through a research and development manager, a local research and development committee, and the research governance framework. The governance framework sets out the various responsibilities. For example, the governance framework for the NHS in Wales ${ }^{10}$ identifies the responsibilities of the participant, the researchers, the principle investigator, funders, the sponsor, employing organisations, care organisations, the responsible care professional, and the research ethics committee. The principle investigator is responsible for ensuring that the chief executive of the care organisation involved in the research is informed and that "their approval is given before the research commences".

The research sponsor is "the organisation taking primary responsibility for ensuring that the design of the study meets appropriate standards and that arrangements are in place to ensure appropriate conduct and reporting; the sponsor is usually, but does not have to be, the main funder". In some instances the sponsor will be the care organisation. The sponsor is responsible, among other things, "for ensuring the quality of the research environment within which the research will be undertaken and the experience and expertise of the principal investigator and other key researchers involved". It is the responsibility of the organisation providing care to ensure that any research involving their patients, users and carers, or staff meet the standards set out [in the framework], in particular that it has an identified research sponsor willing and able to discharge its responsibilities, and that clear and documented agreements are in place about the allocation of responsibilities between all parties involved. Accountability for this lies with the chief executive or agency director.

Pharmaceutical industry sponsors, in particular, have concerns about investigator competence and probity, given that they are often paying substantial sums to local investigators. This is part of the reason for monitors, inspections, and so on, which have highlighted a number of high profile cases of research fraud. ${ }^{11}$ The local REC has rarely, if ever, had a direct role in this and lacks the resources and the expertise to take on such a role. Similarly, while many pharmaceutical sponsors offer investigator training to ensure the quality of investigator recruitment, the local REC is not placed to make such assessment and actually has never effectively done so.

It would thus seem that, effectively, the responsibility for ensuring the suitability of the local investigator and the research setting rests with the sponsor, the principle investigator, and the care organisation. This has clear advantages. All of these bodies or individuals will have personal knowledge of the researcher, and the opportunity and means to assess his or her competence and suitability. They will also have clear organisational responsibility, accountability, and liability for both the conduct of the researchers and the research in ways that the REC cannot possibly have. The present guidelines, however, would result in duplication of effort. While the Research Governance Framework places the responsibility with the sponsor, the principle investigator and the care organisation, the European directive and GAFREC both charge the ethics committee with responsibility for the suitability of the researcher and the local facilities.

\section{REASSURING THE PUBLIC}

One further role of the local REC may be suggested: that it provides reassurance to the local community of the validity of ethical review. Indeed this is explicitly stated in the ICH GCP guidelines, which state that "one of the responsibilities of the Ethics Committee is...to provide public assurance of that protection". Similarly, in the UK, the Guidelines on the Practice of Ethics Committees in Medical Research from the Royal College of Physicians state: "The objectives [of ethics committees] are...to provide reassurance to the public that this [protection of subjects] is being done ${ }^{\prime \prime} .{ }^{12}$

From this perspective lay members of RECs particularly might be thought to be there to make the committee more democratically representative, more representative of the community as a whole, or more accountable to the community as a whole. Local review may not alter the ethics of a study, but it could be seen to be a useful check, a reassurance that justice is seen to be done. However, even if we believe this function to be necessary, we might nevertheless question whether the local REC can perform it.

To suggest the need for RECs to be responsible for such a check is to suggest that the REC is the only body concerned with the ethics of a study. This is not the case: research sponsors, NHS bodies hosting research, and the investigators themselves all share this responsibility. RECs may have a key role in ethical opinion making but no one suggests that their role is an exclusive one, or that research sponsors and investigators are not responsible for proposing ethical research. The role of the local REC in providing a further check may therefore be no more than 
another obstruction. Not all obstructions are necessarily a bad thing, but given an effective system of main ethical review, a proper governance structure, and the lack of training of many local REC members, it is an obstruction that probably serves no useful purpose and should be avoided.

It is doubtful in any case that REC members are, in any meaningful sense, locally representative. In big cities especially, they often reside outside the catchment areas of the institutions in which the research is to take place. They are not elected and, in the past, have often achieved their place on the REC by invitation. Some-perhaps clergy are a good example-may have excellent community credentials, but others, such as non-executive directors of trusts, have none and represent another voice from the articulate middle classes, who are already well represented by the professional members. If those objections were not sufficient, the restructuring of the NHS, with strategic health authorities in England and the abolition of health authorities in Wales, means that the claim that the LREC can represent a community becomes even more difficult to sustain. It is therefore hard to see how a local REC could give adequate reassurance to the local community beyond that already offered by the central committee. Local REC annual reports are currently the only vehicle of accountability and the profile of the REC is close to invisible in most communities. It is doubtful if most annual reports achieve even a sentence or two in the local newspaper.

It might be argued that a research project could be unethical if it offends in some way against cultural or social norms, which might be locally determined. The debate about cultural relativity in ethics is rather beyond the scope of this paper, but it is generally the case that we respect cultural practices relating to such things as diet, worship, or dress, while not accepting practices such as female circumcision, forced marriage, or the immolation of widows. However these conventions are established at a national level and would be the responsibility of central ethical review, the local investigator, and local governance arrangements. There are also more localised concerns, which may relate to a particular sensitivity in a particular community. A project on some aspects of community paediatrics and child protection, for example, might be thought inappropriate in a small town like Soham where a particularly high profile case of child murder took place in 2002. If this is an ethical objection, it is at a level at least one step removed from research ethics, being more about a general feeling that one should not do something that might cause offence or distress. This would elevate "appropriateness" into an ethical principle to be balanced alongside others, although it is interesting to note that, as far as we are aware, there is no objection to research into the management of trauma in Northern Ireland, or into the after effects of exposure to radiation either from the bombs dropped on Nagasaki and Hiroshima or the nuclear accident at Chernobyl.

A local REC might be thought to be best placed to judge such matters of local acceptability, if it were a truly local and representative body. On the other hand, this sort of reservation could equally apply to an epidemiological study, where there is no local researcher and thus no requirement for local ethical review and so this may be another example where responsibility is best devolved to the research and development structure of a trust or primary care body. The question is not about the ethics of the study protocol itself but about its acceptability at a particular time and place. Such cases are likely to be very rare and hardly justify an elaborate system of review by committee. It should be sufficient simply to build into the review of all protocols a requirement for local investigators to go through local research and development bodies, who should ensure that an awareness of local sensitivities forms part of their decision making. There is no reason to believe that the local REC would be any more knowledgeable of local recent history or other sensitivities, given its unrepresentative nature and the extent of the geographical area it must oversee, so even if such pragmatic issues were to be viewed as "ethical", one can still doubt whether the REC is the most competent body to deal with them.

\section{A WAY FORWARD}

The recruitment of sites is the responsibility of the principal investigator, who is also (with others) responsible for ensuring the suitability of the local researcher and the local facilities. He or she must also ensure that the chief executive of the care organisation responsible for the care of the participants gives his or her approval. The chief executive shares responsibility for ensuring that the local investigator is acceptable and that the resources and facilities are in place, and he or she is liable for any harm that may befall patients in his or her care.

When the principal investigator seeks ethical approval, he or she will include details of the sites and local researchers recruited so far with the application. If the principal investigator is required to submit that information together with a signed statement from the respective chief executives to the effect that the research has been approved within the research governance framework for the care organisations, the REC reviewing the proposal will know that a properly responsible and accountable body has accepted responsibility and liability for the local researcher and research facilities in each site. Thus the study would need no further review beyond the main ethical review by the REC. Sites and local investigators recruited after ethical approval had been received would be notified to the REC, with the letter of governance approval from the chief executive. As an aside, we note that because RECs are advisory bodies only (and with respect to ethics, not monopoly ones), it is possible in principle that the research and development committee or even the chief executive could prevent the study locally on ethical grounds, as well as on grounds of research governance.

For research in NHS hospital trusts this arrangement would be relatively straightforward. In primary care, where general practitioners (GPs) conduct research either as principal or local investigators, it may not be quite so clear. The requirements of the governance framework are clear enough, but it is not immediately obvious as to who should function in the various roles, as the GP researcher may also be the chief executive of the care organisation and in some instances might also be the sponsor. However, the GP's patients are under his or her care by virtue of the contract to provide general medical services, and thus it would seem reasonable for the body that controls and monitors this contract to accept responsibility for the research activities of GPs and monitor their performance; in Wales for example this would be the local health board. In both the hospital and the primary care settings there is also the possibility of conflicts of interest. Research, particularly when funded by the pharmaceutical industry, may generate considerable income for the local investigator, and a successful research career may also bring considerable prestige and professional advancement for the researcher. Hospital trusts may rely on research income to subsidise clinical services, members of staff may depend on it for their continued employment, and GP practices may depend on it to fund posts or other activities. However the governance structure, while not guaranteeing that there will be no abuses of the system, does at least provide a system of regulation and accountability through 
which action can be taken should malpractice be identified.

\section{SUMMARY AND CONCLUSIONS}

We have argued in this paper that the only questions of research ethics relating to studies reviewed by RECs are central issues: the REC reviews the ethics of the study. It would be unethical to allow incompetent researchers to conduct research in inadequate facilities, but this is not a question of the ethics of research. The competence of the investigator and the suitability of the facilities are empirical questions that the REC is not competent to address and these are issues that should be outside the responsibility of the REC. The responsibility for the management and conduct of research rests with the sponsor, the principal investigator, and the care organisation and this is well described in the Framework for Research Governance.

The locality issues set out in GAFREC, of the competence of investigators and suitability of facilities, should thus be addressed through the governance framework and not through the REC system. Applicants for ethical review should present their protocols with details of sites and local researchers already approved by the relevant authority through governance procedures, and the convention of local review by the REC should be abandoned. Research ethics committees should, however, be notified of research to be conducted in their area, as they are with current studies where there is no local researcher. As part of the main ethical review, RECs should satisfy themselves that there has been proper scrutiny of the project through the local research governance framework in each of the proposed sites and that the appropriate local manager has accepted responsibility for the conduct of the research. The requirement set out in the European directive for RECs to "consider" such issues as the suitability of the local investigator and quality of facilities can thus be fulfilled by scrutiny of the local governance agreement. This also fulfils the requirements of the Directive that protocols undergo ethical review by a single body.

There may be some concern that governance arrangements are in their infancy and not yet sufficiently robust to provide sufficient reassurance of the protection of the research participant. If this is the case, the problems should be addressed by strengthening the governance system. After all, it is the sponsor, principal investigator, and care organisation who are responsible for the conduct of the research and the safety of all involved and who will be held accountable and liable in the event of any damage to research participants.

J Med Ethics 2004;30:313-317.

doi: 10.1136/jme.2003.004051

Authors' affiliations

Authors' affiliations

P Wainwright, Centre for Philosophy,

Humanities and Law in Health Care, University of Wales, Swansea, UK

J Saunders, Centre for Philosophy, Humanities and Law in Health Care and Nevill Hall Hospital, Abergavenny, UK

Dr Saunders is Chairman, and Dr Wainwright is a member, of the Multi-Centre Research Ethics Committee for Wales. However the views expressed in this article are personal and do not represent the views of the Committee, or of the National Assembly for Wales.
Correspondence to: P Wainwright, Centre for Philosophy, Humanities and Law in Health Care, University of Wales Swansea, Singleton Park, Swansea SA2 8PP, UK;

p.wainwright@swansea.ac.uk

Received 17 March 2003

Revised 27 June 2003

Accepted 1 July 2003

\section{REFERENCES}

1 Department of Health. Local Research Ethics Committees, Health Service Guidelines HSG(91)5, London, 1991.

2 Evans D, et al. Report to the Department of Health on the conduct of ethical review of multi-location research involving human subjects. Swansea: Centre for Philosophy and Health Care, University College, 1992

3 NHS Executive. Ethics committee review of multicentre research. HSG(97)23, April 1997

4 European Draft Clinical Trials Directive 2001/ 20/EC (short title). Official Journal of the European Communities, May 2001.

5 DoH Governance arrangements for NHS Research Ethics Committees. London: Department of Health, July 2001

6 European Commission Enterprise Directorate. General detailed guidelines on the principles of good clinical practice in the conduct in the EU of clinical trials on medicinal products for human use. Brussels, July 2002.

7 DoH Supplementary Operational Guidelines for NHS Research Ethics Committee: Multi-centre research in the NHS - the process of ethical review when there is no local researcher. London: Department of Health, 2000.

8 Megone C, Mason SA, Allmark PD, et al. The structure, composition and operation of European Research Ethics Committees. In Mason S Megone C, eds. European Neonatal Research Consent, ethic committees and law. Aldershot: Ashdown, 2001:23-42.

9 Council of Europe Survey of the Procedures for Ethical Review in the Council of Europe Member States, Strasbourg, January 1998.

10 National Assembly for Wales Research Governance Framework For Health And Social Care In Wales Cardiff, 2001.

11 Smith R. Time to face up to research misconduct [editorial]. BMJ 1996;312:789-90.

12 Royal College of Physicians. Guidelines on the Practice of Ethics Committees in Medical Research Involving Humans. Third edition. London: Royal College of Physicians of London, 1997. 